\title{
Strength and Power Changes During an In-season Resistance Training Program for Male CIS Volleyball Players
}

\author{
Doug Hillis, Matthew Okrainec*
}

\begin{abstract}
Previous research has indicated that performing repetitions to failure at greater than $80 \%$ one repetition max (1 RM) one day per week using a non-linear training program can maintain strength and power in many athletic populations. However, this finding has not been directly assessed in male university volleyball players. The purpose of this study was to determine if non-linear training two days a week could maintain upper and lower body strength, and vertical jump power and height over the course of a competitive volleyball season, measured at three time points. Eleven trained Canadian Interuniversity Sport (CIS) male athletes were a part of a single training group which completed a maintenance program consisting of non-linear training for 17 weeks. At each testing time point, the participants completed a bench press, squat, squat jump, countermovement jump, and three step jump and reach. We predicted that non-linear resistance training of mixed intensities twice a week would allow players to maintain strength, vertical jump power, and vertical jump height. Results indicated that upper body strength can be maintained over the course of a competition period with non-linear resistance training $(p=0.043)$. Scores for lower body strength, vertical jump squat jump power, vertical jump countermovement jump power, and vertical jump height increased over the course of the season ( $p$ 's $<0.001,0.14,0.181$, and 0.006 , respectively). Our results indicate that male volleyball players can benefit from non-linear training to maintain or improve performance when training at $>80 \%$ I RM to failure once a week during a competition season.
\end{abstract}

Keywords: non-linear training, volleyball, maintenance, strength, power

\section{Introduction}

Resistance training programs are typically designed to help athletes become more powerful, and more effective in their given sport. Athletes can build towards a career of optimal performance and injury prevention if developing athletes are able to maintain or increase gains made during the off- season throughout the in-season competition. Creating a team program for college players involves accounting for what will be safe and appropriate for all athletes but will allow athletes to progress or maintain progress from the off-season. Recent research has focused primarily on intensity as the most important factor for maintaining strength and power throughout the in-season, since frequency of training is usually reduced from three to four

\footnotetext{
* College of Kinesiology, University of Saskatchewan, Saskatoon, SK, Canada

Correspondence: mro814@mail.usask.ca
} 
days to two days per week (Hoffman, 2002; Hoffman \& Kang, 2003; Cardosa Marques, Gonzales-Badillo, \& Kukla, 2006). Discontinuing resistance training after experiencing performance adaptations leads to decreases in strength and power over the course of the competition period, which is referred to as detraining (Marques, Zajac, Pereira, \& Costa, 2011). In terms of vertical jump height, detraining can affect individuals differently depending on the population. Sportspecific training will ultimately determine how an athlete's vertical jump height may change if exposed to prolonged detraining periods with no resistance training. Athletes can maintain jump height even with loss of overall strength and power if enough specific jumps are performed during detraining periods.

Research has suggested that without heavy resistance training to failure or near failure at least once a week, an athlete's strength and power could decrease significantly (Marques, Tillar, Vescovi, \& Gonzalez-Badillo, 2008). Significant losses of strength and power from a lack of heavy training over the course of a season can become evident within as few as four weeks (Marques et al., 2011). Although lifting heavier loads once a week may have important benefits, it is also necessary to take precautions against potential injury or excessive muscle soreness. An appropriate repetition range must be chosen that suits the experience of the players on the team to avoid this, as performing exercises with heavy loads at low reps can be difficult. Individuals should aim for a minimum of six repetitions to prevent or diminish the possibility of muscle soreness, damage, and injury (Arazi \& Asadi, 2013). Research has indicated that athletes may be able to maintain strength and power throughout a competitive season for some sports when working with loads approximately 80 to $85 \%$ one repetition $\max (1 \mathrm{RM})$ at six to eight repetitions for heavy intensity days (Hoffman, 2002; Hoffman \& Kang, 2003; Hoffman, Wendell, Cooper, \& Kang, 2003; Baechle \& Earle, 2008). An individual's 1 RM is the maximum amount of load that can be successfully lifted for one maximal repetition. Lifting at loads of 80 to $85 \% 1$ RM for heavy days will still allow high velocity during the lift, which is highly beneficial for the skills used in volleyball (Holmberg, 2013). It is important to complete a non-linear training program to get an appropriate mix of training to maintain or improve performance qualities, while not hindering performance during the in-season, where there are multiple games in a week (Cardosa Marques et al., 2006). Non-linear training involves training at different intensities during the week, including lighter intensities without lifting to failure to ensure game day recovery. Cardosa Marques et al. (2006) suggests that non-linear training allows athletes to perform heavy resistance training and maintain off-season gains, while also completing other sessions that will not create soreness and fatigue, which otherwise could be detrimental to performance on game days.

The purpose of this study was to examine whether performing non-linear training two days a week could maintain upper and lower body strength and vertical jump power and height throughout a college volleyball competitive season. It was hypothesized that non-linear resistance training of mixed intensities twice a week would maintain or increase strength, vertical jump power, and vertical jump height in Canadian Interuniversity Sport (CIS) male athletes.

\section{Methods}

\section{Participants and Prior Training}

The participants for this study included 11 male CIS volleyball players from the University of Saskatchewan. The 11 participants consisted of all positions including outside hitter, middle, setter, and libero. Due to injury, only 10 participants could be analyzed for predicted 1 RM for bench press and only seven participants could be analyzed for predicted 1 RM for squat, predicted peak vertical jump power values for squat jump and countermovement jump, and vertical jump height for the three step jump and reach. For this study, participants were considered trained if they had at least one year of experience with post-secondary volleyball programs and resistance training. Those on the team not considered trained were not eligible to participate in the study. The participants selected for this study were already very familiar with resistance training and testing procedures. Untrained participants were excluded to eliminate any major confounding factors from learning. The amount of time spent training per week changed multiple times during the study between pre-season and competitive season periods, and the CIS Christmas break, explained in further detail in Table 1. During the pre-season, participants trained three days a week for eight weeks. Participants trained two days a week for six weeks during the first part of the regular season, and then three days a week during the CIS Christmas break period (four weeks). Participants again trained two days a week for the final seven weeks during the second half of the regular season. Training targeted the upper and lower body and incorporated exercises targeting large muscle groups. Bench presses and squats were the testing focus and were incorporated into the pre-season training workouts. Training sessions were supervised whenever possible and when supervision was not available, attendance was taken to ensure workouts were completed.

\section{Experimental Design}

Testing for the research study was conducted at three time points:

University of Saskatchewan Undergraduate Research Journal 
1) At the beginning of the competitive season (preseason)

2) At the beginning of the second half of the regular season (mid-season)

3) At the end of the competitive season (post-season) These time points are further illustrated in Table 1 . The participants completed resistance training two days a week, in addition to volleyball-specific practices, scheduled between the three time points. The testing protocol at each time point included predicted 1 RM bench press and 1 RM squat, predicted power from a squat jump, predicted power from a countermovement jump, and a three step jump and reach test.

Table 1: A timeline representing the experimental design of the study outlining the three testing time points and the corresponding frequency of resistance training that was completed between each testing.

\begin{tabular}{|c|c|c|}
\hline 1 & 2 & 3 \\
\hline October 28 & January 6 & February 25 \\
\hline Pre-season testing & Mid-season testing & Post-season testing \\
\hline Resistance training & Resistance training & Resistance training \\
\hline completed 3x/week & completed 2x/week & completed $2 x /$ week \\
\hline for 8 weeks & for 10 weeks $^{a}$ & for 7 weeks \\
\hline
\end{tabular}

a resistance training completed $3 x /$ week for 4 weeks of the 10 week timeframe due to the CIS Christmas break

\section{Testing Protocol}

Bench press and squat. Proper technique for the bench press was defined as a lift performed supine on the bench with shoulders and hips always remaining in contact with the bench. The weighted bar was to be lowered from full elbow extension until the bar touched the chest, and the weight was lifted back to full elbow extension to complete one repetition. The thumbs on the bar remained over the lateral part of the shoulders. The movement was to be controlled and no bouncing off of the chest was allowed (LeSuer, McCormick, Mayhew, Wasserstein, \& Arnold, 1997).

Proper technique for the squat was defined as standing with both feet parallel and the inside of the foot lined up with the outside of the shoulder. A weighted barbell was placed on the shoulders behind the neck. The hands were placed on the bar for control. The participants started from full knee extension and bent at the knees until the thighs were parallel with the ground. The investigator of the test then said "up" to the participant when thighs were parallel to the ground (LeSuer et al., 1997).

The testing for the bench press and squat was always performed with at least one day of rest between competitions. There was a minimum of five minutes of rest for recovery between testing for the bench press and squat. De Salles et al. (2009) found that a three to five minute rest interval is necessary for recovery when performing work to failure. The players were asked to pick a weight for each exercise at which they believed they would fail at between six to eight repetitions. The participants picked their weight based on previous resistance training and this weight was approved by the investigator. If the participant successfully completed more than ten repetitions, they were required to rest five minutes and complete the test again with a higher weight. The estimated 1 RM was used to avoid the potential of injury occurring. The Wathan formula (1) was chosen to calculate the predicted $1 \mathrm{RM}$ as it has been shown to have high correlation coefficients between predicted and achieved 1 RM strength ( $r>0.95$ ) (LeSuer et al., 1997).

Vertical jumps. The squat jump movement was defined as starting with bent knees at 90 degrees and the arms behind the individual. The individual had to be static before jumping. The individual attempted the jump when ready, extending straight up from the static position with arms being allowed to be thrust forward (Sayers, Harackiewicz, Harman, Frykman, \& Rosenstein, 1999). The countermovement jump started with the individual in a standing position and when ready, the individual dropped into a crouch position and without pausing, jumped up as high as possible. The individual was allowed to use his arms for momentum in this test. The squat jump and countermovement jump were measured with a mat and belt. A belt attached to the participant's waist with a measuring tape grounded to the mat measures vertical jump height accurately as shown by Buckthorpe, Morris, and Folland (2012). The Sayers equation has been shown to be an accurate formula for predicting peak power and is appropriate for both a squat jump and countermovement jump. Cross-validation of regression equations using the PRESS statistic reveals accurate and reliable values $\left(R^{2}=\right.$ 0.88, SEE = 372.9 W) (Sayers et al., 1999).

For the vertical three step jump and reach test, each individual had his standing reach height recorded. The individual stood fully erect with the feet together flat on the ground and reached as high as possible with the head and eyes level to measure standing height. A Vertec apparatus was used to measure the three step jump and reach. The Vertec apparatus is a steel frame with horizontal vines that are rotated out of the way by the participants to measure the maximal three step jump and reach height. Leard et al. (2007) showed the Vertec to be an accurate measure of vertical jump height for the three step jump and reach test when assessed for concurrent validity with a three-camera video system $(r=0.91)$. To perform the vertical three step jump and reach, the participants were instructed to execute a standard volleyball approach and reach as high as 
possible. A standard volleyball approach was defined as two strides, then bringing the feet together and jumping up off of both feet.

The testing for the three different jump tests were also performed with at least one day of rest between competitions, but on the same day as the bench press and squat. Between each jump test there was a minimum of 15 seconds of rest to recover for the next jump. Read and Cisar (2001) found that a 15 second rest between maximal vertical jumps was sufficient to fully recover for another maximal jump. The players were asked to give a full effort on each jump. A practice trial was allowed for participants to get a feel for the test. Two trials were performed and recorded for the squat jump and countermovement jump. Participants were given 3 trials to move vines on the Vertec for the three step jump and reach test. If an individual moved vines on the third trial, he was given more trials until he could no longer move more vines on the Vertec. The best trial was taken by the investigator and used in the Sayers power prediction equation (Sayers et al., 1999).

\section{In-season Training}

After testing at the first time period (i.e. the preseason time point), participants performed the in-season lifting procedures. Each player received workouts for each weight training day and a log book to keep track of the loads used. Exercises were large, multi-joint exercises, with two of the exercises always being bench press and squat. Other exercises, accessory lifts, varied from workout to workout to target an abundance of muscle groups. A three set protocol was completed for each session for approximately ten to twelve exercises. On the first workout of the week, lifts were performed for six to eight repetitions with at 80 to $85 \%$ I RM until failure or near failure. For the second workout of the week, participants were asked to do a load for six to eight repetitions, but this time to finish their repetitions knowing they still could have done at least two to three more. Training during the Christmas break still involved training with heavy loads to failure once a week, even though training was increased to three days a week during this time. On the third workout of the week during the Christmas break, the training design was similar to the second workout of the week, but the accessory exercises were varied. Pre-season training was also done three times a week with a periodized approach to increase strength and power. These pre-season sessions included multiple days of working at high intensities to failure. Lifting during all repetitions for the study was instructed to be done at a high velocity as long as control of the load was maintained. Telling participants to aim for a load where they could complete a certain amount of repetitions allowed participants not to have to calculate 1 RM for every exercise. Two minutes of rest minimum were given between the same exercises.

\section{Data Analysis}

The Wathan formula was used to obtain a predicted 1RM for the squat and bench press (LeSuer et al., 1997).

(1)

$$
\begin{aligned}
1 \mathrm{RM}(\mathrm{lbs})= & {[100 \times \text { weight lifted }(\mathrm{lbs})] /[48.8+} \\
& \left.53.8\left(\mathrm{e}^{-0.075 \times \text { repetitions }}\right)\right]
\end{aligned}
$$

To obtain peak predicted power for the squat jump and countermovement jump, the Sayers formula was used (Sayers et al., 1999).

(2)

Peak Power $(\mathrm{W})=[60.7 \times$ jump height $(\mathrm{cm})]+$ [45.3 x body mass $(\mathrm{kg})]-2055$

The three step vertical jump values were analyzed as the raw vertical height scores and were not used in the Sayers formula. A series of one-way repeated measures analysis of variance (ANOVA) on the pre-, mid-, and post-season scores were used with time point as the independent variable. Significance was set at $p<0.05$ and post-hoc analysis was performed using $95 \%$ confidence intervals according to the Loftus and Masson (1994) method.

\section{Results}

Table 2: Comparison of the mean, standard deviation, and $p$ values of the measured testing parameters at pre-, mid-, and post-season testing times.

\begin{tabular}{lllll}
\hline Testing parameter & P value & $\begin{array}{l}\text { Pre (Pre- } \\
\text { Mean } \pm \text { SD) }\end{array}$ & $\begin{array}{l}\text { Mid (Mid- } \\
\text { Mean } \pm \text { SD) }\end{array}$ & $\begin{array}{l}\text { Post (Post- } \\
\text { Mean } \pm \text { SD) }\end{array}$ \\
\hline BodyMass $(\mathrm{kg})$ & .576 & $87.8 \pm 8.5$ & $87.4 \pm 8.5$ & $87.6 \pm 8.0$ \\
Bench Press $(\mathrm{kg})$ & $.043^{\mathrm{bc}}$ & $97.7 \pm 14.1$ & $97.1 \pm 13.3$ & $100.1 \pm 12.3$ \\
Squat $(\mathrm{kg})$ & ${<.001^{\mathrm{abc}}}$ & $135.9 \pm 11.1$ & $146.0 \pm 12.0$ & $151.4 \pm 11.3$ \\
Squat Jump (W) & $.014^{\mathrm{ab}}$ & $5770.5 \pm$ & $6052.3 \pm$ & $6145.2 \pm$ \\
& & 393.5 & 365.4 & 421.4 \\
Countermovement & .181 & $5770.50 \pm$ & $6052.3 \pm$ & $6145.2 \pm$ \\
Jump (W) & & 393.5 & 365.4 & 421.4 \\
Jump \& Reach (cm) & $.006^{\text {ab }}$ & $78.3 \pm 7.6$ & $81.6 \pm 6.6$ & $82.6 \pm 7.9$ \\
\hline
\end{tabular}

a significant difference between pre-season and mid-season testing time points.

${ }^{b}$ significant difference between pre-season and postseason testing time points.

c significant difference between mid-season and postseason testing time points. 


\section{Discussion}

These results show that strength, vertical jump power, and vertical jump height were able to be maintained, and in some cases even increased, over the course of the 17 week competition period. All participants increased $1 \mathrm{RM}$ predicted scores across all time points for the squat exercise. Strength was maintained from pre-season to midseason and then increased from mid-season to post-season for the 1 RM bench press. In terms of vertical jump power, the scores for predicted power in the squat jump increased at mid-season and post-season from the initial pre-season data. The predicted power for the countermovement jump was maintained throughout the season. With regard to vertical jump height, the scores for the three step jump and reach test were increased at mid-season and post-season from the initial pre-season data.

In terms of strength these results suggests that a nonlinear maintenance program for university CIS volleyball athletes that involves lifting to failure at an intensity of > $80 \%$ I RM once a week has the ability to increase upper and lower body strength throughout the course of the competition period. Our study supports previous research that indicates that performing exercises at > 80\% 1 RM can be used to maintain or increase strength throughout a season, especially in the lower body (Hoffman \& Kang, 2003; Marques et al., 2008; Ronnestad, Nymark, \& Raastad, 2011; Baker, 2001). The greater increases in the squat compared to the bench press could be attributed to the fact that volleyball players are constantly jumping and using their legs throughout the season during practices and games. This is consistent with previous research which states that sports involving mass amounts of jumping can elicit greater strength gains in the lower body (Robertson et al., 2001).

With regard to vertical jump power and vertical jump height, this study supports previous research indicating that vertical jump power and height can be maintained or increased throughout a season for volleyball athletes (Robertson et al., 2001; Marques et al., 2008; Ronnestad et al., 2011). Our study also supports previous research indicating that activation of lower body musculature (focused primarily on explosive movements) through resistance training programs during in-season competition has the ability to enhance power and jumping performance (Comfort, Stewart, Bloom, \& Clarkson, 2014). Therefore, it is possible that throughout a competition period, especially in earlier parts of the season, athletes can increase their vertical jump power and height as they continue to practice sport specific skills while participating in a resistance training program.

Our results also indicate that an intensity of 8 oto $85 \%$ $1 \mathrm{RM}$ to failure once a week can maintain or benefit a male
CIS volleyball player's performance throughout the season. Increases or maintenance of the specific testing criteria in our study indicate that the reduction in frequency of training to two days from three days a week during the competition season can still elicit strength, power, and vertical height improvements. The use of a non-linear training program also attempts to prevent fatigue, soreness, and other detriments for the best possible game day performance (Cardosa Marques et al., 2006). By reducing frequency and maintaining an above $80 \%$ intensity, athletes can maintain their off-season improvements while also ensuring that they feel prepared for competition.

Though we did find that male CIS volleyball players were able to maintain or increase strength and power through the competitive season, there were still some limitations to the study. There was no control group because it was thought that those who were not offered the resistance training could have had their performance for the season affected. It would be of interest to know whether a control group would be able to maintain strength and power through the season for the testing protocol if only exposed to practices and games. It is also possible that the amount of practices and games performed by the participants contributed to their improved performance in strength and jumping tests. The participants were usually competing in either volleyball practices or games six days a week. This extra overload from constant jumping and sport specific activity, including constant jumping, could have contributed to the results of the testing throughout the season. Therefore, it cannot be conclusively said that the resistance training program in place was the sole reason for the maintenance or increase of strength and power. Another important consideration was the Christmas break for four weeks between the pre-season and mid-season time points. During this time, the players were allowed to lift three times a week instead of two, although the players still only lifted to failure once a week for 80 to $85 \% 1$ RM. It is important to note that some of the increases or maintenance between the pre-season and mid-season time points could be attributed to this increased frequency of lifting during Christmas break. Another limitation is that players were not supervised during one of the resistance training sessions during the week. It would have been beneficial to have a researcher supervising every session throughout the year in order to ensure that the training regimen was being followed to the specifications of the program designed by the researcher throughout the season. Lastly, the squat and bench press exercise were the only exercises that remained constant for every resistance training session throughout the season, so we cannot determine which exercises were specifically able to maintain strength and power. 


\section{Conclusion}

The results from this study provide support for the effectiveness of non-linear resistance training to maintain or increase strength and power throughout a competition season. Seeing increases throughout the season in strength, power, and vertical height is ideal, but maintenance should be the minimum players strive for. Our results suggest that non-linear training with one day of heavy intensity training a week has the ability to maintain, and in some cases even increase, the strength and power of developing athletes over a competitive season. Maintaining or increasing the gains made during the off-season during in-season competition allows developing athletes to build towards a career of optimal performance and injury prevention.

Future research on the topic of non-linear training during a competitive season should focus on different playing positions within a volleyball team. Different positions in the game of volleyball require different body types and physical demands, which could change the effect resistance training has on each position. It would also be beneficial to look at different measures for upper body strength and power. In this study the bench press criterion was not very specific to volleyball, so a more sport-specific measurement would be of interest to examine. It would be ideal to include a measure specific to volleyball upper body power. Looking at subjective perceived soreness for the players could also provide insight on the effectiveness of non-linear training. Since the goal of non-linear training is to enhance recovery it would be interesting for players to subjectively perceive their level of recovery before participation on game days. Further, research should also look at the asymmetries of volleyball players who participate in resistance training opposed to those who do not, as muscle asymmetries can put an athlete at a greater risk of injury (Hadzic, Sattler, Veselko, Markovic, \& Dervisevic, 2014). In volleyball, one side of the body may be more dominant than the other, specifically the upper body because of attacking. It would be of interest to study the effects of non-linear resistance training on these asymmetries. 


\section{References}

Arazi, H., \& Asadi, A. (2013). One repetition maximum test increases serum indices of muscle damage and soreness in trained and untrained males. Apunts. Medicina de l'Esport, 48(178), 49-54. http://dx.doi.org/10.1016/j.apunts.2012.09.003

Baechle, T. R., \& Earle, R. W. (Eds.). (2008). Essentials of strength training and conditioning. Champaign, IN: Human kinetics.

Baker, D. (2001). The effects of an in-season of concurrent training on the maintenances of maximal strength and power in professional and college aged rugby league football players. Journal of Strength and Conditioning Research, 15(2), 172-177. Retrieved from https://www.antoniocgomes.com/wpcontent/uploads/2012/10/HAGBY.pdf

Bernhardt, D. T., Gomez, J., Johnson, M. D., Martin, T. J., Rowland, T. W., Small, E., Leblanc, C., Malina, R., Krein, C., Young, J. C., Reed, F. E., Anderson, S. J., Griesemer, B. A., Bar-or, O. (2001). Strength training by children and adolescents. Pediatrics, 107(6), 1470-1472. doi: 10.1542/peds.2007-3790

Brooks, G. A., Fahey, T. D., \& Baldwin, K. M. (2005). Exercise physiology: Human bioenergetics and its applications (4th Ed.). Boston, MA: McGraw-Hill.

Buckthorpe, M., Morris, J., \& Folland, J. P. (2012).Validity of vertical jump measurement devices. Journal of Sports Sciences, 30(1), 63-69. doi: $10.1080 / 02640414.2011 .624539$

Cardosa Marques, M. A., Gonzales-badillo, J. J., \& Kukla, D. A. (2006). In-season resistance training for professional male volleyball players. Strength and Conditioning Journal, 28(6), 16-27. doi: 10.1519/00126548-200612000-00002
Cardosa Marques, M., \& Gonzalez-badillo, J. J. (2006). Inseason resistance training and detraining in professional team handball players. Journal of Strength and Conditioning Research, 20(3), 563-571. Retrieved from http://search.proquest.com/docview/213063162?acc ountid=14739

Carron, A. V., \& Spink, K. S. (1993). Team building in an exercise setting. Sport Psychologist, 7, 8-18. doi:10.1080/10413209708415384

Comfort, P., Stewart, A., Bloom, L., \& Clarkson, B. (2014). Relationships between strength, sprint, and jump performance in well-trained youth soccer players. Journal of Strength and Conditioning Research, 28(1), 173-177. doi: 10.1519/JSC.obo13e318291b8c7

De Salles, B. F., Simao, R., Miranda, F., Silva Novaes, J., Lemos, A., \& Willardson. J. M. (2009). Rest interval between sets in strength training. Sports Medicine, 39(9), 765-777. doi: 10.2165/11315230000000000-00000

Faigenbaum, A. D., \& Myer, G. D. (2010). Resistance training among young athletes: safety, efficacy and injury prevention effects. British Journal of Sports Medicine, 44(1), 56-63. doi: 10.1136/bjsm.2009.0680g8

Faigenbaum, A. D., Westcott, W. L., Micheli, L. J., Outerbridge, A. R., Long, C. J., Larosa-loud, R., \& Zaichkowskt, L. D. (1996). The Effects of Strength Training and Detraining on Children. Journal of Strength Conditioning Research, 10(2), 109-114. doi: $10.1177 / 1941738109334215$

Hadzic, V., Sattler, T., Veselko, M., Markovic, G., \& Dervisevic, E. (2014). Strength asymmetry of the shoulders in elite volleyball players. Journal of Athletic Training, 49(3), 338. doi: 10.4085/1062-605049.2.05

Hakkinen, K. (1993). Changes in physical fitness profile in female volleyball players during the competitive season. The Journal of Sports Medicine and Physical Fitness, 33(3), 223-232. 
Hoffman, J. (2002). Physiological aspects of sport training and performance. Champaign, IL: Human Kinetics.

Hoffman, J., \& Kang, J. (2003). Strength changes during an in-season resistance-training program for football. Journal of Strength and Conditioning Research, 17(1), $109-114$.

Hoffman, J., Wendell, M., Cooper, J., \& Kang, J. (2003). Comparison between linear and nonlinear in-season training programs in freshman football players. Journal of Strength and Conditioning Research, 17(3), 561-565.

Holmberg, P. A. (2013). Weightlifting to improve volleyball performance. Journal of Strength and Conditioning Research, 35(2), 79-88. doi: 10.1519/SSC.obo13e318288gf 47

Hortobagyi, T. T., Houmard, J. A., Stevenson, J. R., Fraser, D. D., Johns, R. A., \& Israel, R. G. (1993). The effects of detraining on power athletes. Medicine and Science in Sports and Exercise, 25(8), 929-935. DOI: 10.1249/00005768-199308000-00008

Kraemer, W. J., Patton, J. F., Gordon, S. E., Harman E. A., Deschenes, E. R., Reynolds, K., Newton, R. U., Triplett, N. T., \& Dziados, J. E. (1995). Compatibility of high-intensity strength and endurance training on hormonal and skeletal muscle adaptations. Journal of Applied Physiology, 78(3), 976-989. Retrieved from http://jap.physiology.org/content/78/3/976

Kraemer, W. J., Ratamess, N., Fry, A. C., Triplett-McBride, T., Koziris, L. P., Bauer, J. A., Lynch, J. M., \& Fleck, S. J. (2000). Influence of resistance training volume and periodization on physiological and performance adaptations in collegiate women tennis players. American Journal of Sports Medicine, 28(5), 626-633. Retrieved from http://ajs.sagepub.com/content/28/5/626.long
Leard, J. S., Cirillo, M. A., Katsnelson, E., Kimiatek, D. A., Miller, T. W., Trebincevic, K., \& Garbalosa, J. C. (2007). Validity of two alternative systems for measuring vertical jump height. Journal of Strength and Conditioning Research, 21(4), 1296-1299. doi: 10.1519/R-21536.1

Legg, D., \& Burnham, R. (1999). In-season shoulder abduction strength changes in football players. Journal of Strength and Conditioning Research, 13(4), 381-383. doi: 10.1097/00124278-199911000-00013

LeSuer, D. A., McCormick, J. H., Mayhew, J. L., Wasserstein, R. L., \& Arnold, M. D. (1997). The accuracy of prediction equations for estimating 1-RM performance in the bench press, squat, and deadlift. Journal of Strength and Conditioning Research, 11(4), 211-213.

Linnamo, V., Hakkinen, K., \& Komi, P. V. (1998). Neuromuscular fatigue and recovery in maximal compared to explosive strength loading. European Journal of Applied Physiology and Occupational Physiology, 77(1), 176-181. Retrieved from http://link.springer.com.cyber.usask.ca/article/10.10 $07 / 5004210050317$

Loftus, G. R. \& Masson, M. E. (1994). Using confidence intervals in within-subject designs. Psychonomic Bulletin and Review, 1(4), 476-490. doi: 10.3758/BFo3210951

Markovic, G., Dizdar, D., Jukic, I., \& Cardinale, M. (2004). Reliability and factorial validity of squat and countermovement jump tests. The Journal of Strength and Conditioning Research, 18(3), 551-555.

Marques, M. C., Tillar, R. V., Vescovi, J. D., \& Gonzalezbadillo, J. J. (2008). Changes in strength and power performance in elite senior female professional volleyball players during the in-season: a case study. Journal of Strength and Conditioning Research, 22(4), 1147-55. doi: 10.1519/JSC.obo13e31816a42do 
Marques, M. C., Zajac, A., Pereira, A., \& Costa, A. M. (2011). Strength training and detraining in different populations: Case studies. Journal of Human Kinetics, 29A, 7-14. doi: 10.2478/v10078-011-0052-7

Neufer, P. D., Costill, D. L., Fielding, R. A., Flynn, M. G., \& Kirwan, J. P. (1987). Effect of reduced training on muscular strength and endurance in competitive swimmers. Medicine and Science in Sports and Exercise, 19(5), 486-490.

Newton, R. U., Hakkinen, K., Hakkinen, A., McCormick, M., Volek, J., \& Kraemer, W. J. (2002). Mixed-methods resistance training increases power and strength of young and older men. Medicine and Science in Sports and Exercise, $34(8), 1367-1375$.

Rashad, A. K., (2011). Comparing two methods of maintaining specific physical fitness abilities level during the volleyball junior's competitive season. World Journal of Sport Sciences, 4(2), 98-103. Retrieved from http://www.idosi.org/wjss/4\%282\%2911/3.pdf

Read, M. M., \& Cisar, C. (2001). The influence of varied rest interval lengths on depth jump performance. Journal of Strength and Conditioning Research, 15(3), 279283.

Robertson, K. M., Newton, R. U., Doan, B. K., Rogers, R. A., Shim, J., Popper, E. M., Horn, B., Hakkinen, K., \& Kraemer, W. J. (2001). Effects of in-season strength and power training on squat jump performance in NCAA women volleyball players. Medicine and Science in Sports and Exercise, 33(5), S134. doi: 10.1097/00005768-200105001-00764

Ronnestad, B. R., Nymark, B. S., \& Raastad, T. (2011). Effects of in-season strength maintenance training frequency in professional soccer players. Journal of Strength and Conditioning Research, 25(10), 2653-60. doi: 10.1519/JSC.obo13e31822dcdg6
Rontu, J. P., Hannula, M. I., Leskinen, S., Linnamo, V., \& Salmi, J. A. (2010). One-repetition maximum bench press performance estimated with a new accelerometer method. Journal of Strength and Conditioning Research, 24(8), 2018-25. doi: 10.1519/JSC.obo13e3181c7C433

Sanborn, K., Boros, R., Hruby, J., Schilling, B., O’Bryant, H. S., Johnson, R. L., Hoke, T., Stone, M. E., \& Stone, M. H. (2000). Short-term performance effects of weight training with multiple sets not to failure vs. a single set to failure in women. Journal of Strength and Conditioning Research, 14(3), 328-331. Retrieved from http://www.readcube.com/articles/10.1097\%2Fo012 4278-200008000-00014

Sayers, S. P., Harackiewicz, D. V., Harman, E. A., Frykman, P. N., \& Rosenstein, M. T. (1999). Cross-validation of three jump power equations. Medicine and Science in Sports and Exercise, 31(4), 572-577.

Schneider, V., Arnold, B., Martin, K., Bell, D., \& Crocker, P. (1998). Detraining effects in college football players during the competitive season. Journal of Strength and Conditioning Research, 12(1), 42-45. Retrieved from http://www.readcube.com/articles/10.1097\%2Fo012 4278-199802000-00009 
Strength and Power Changes for In-Season Resistance Training (Okrainec \& Hillis)

University of Saskatchewan Undergraduate Research Journal 